\title{
Sarah Carvallo
}

\section{LA CIRCULATION}

\section{SANGUINE COMME}

\section{PIERRE DE TOUCHE :}

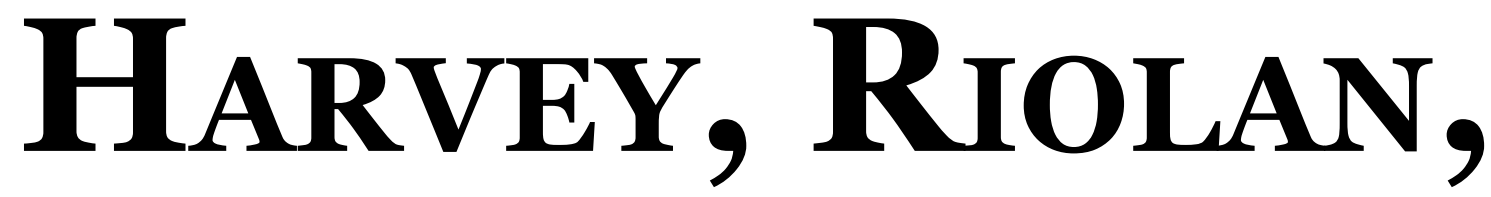

Descartes



SOCIÉTÉ DE PHILOSOPHIE DES SCIENCES (SPS) 
Sarah Carvallo

\section{LA CIRCULATION SANGUINE COMME PIERRE DE TOUCHE : Harvey, Riolan, Descartes}

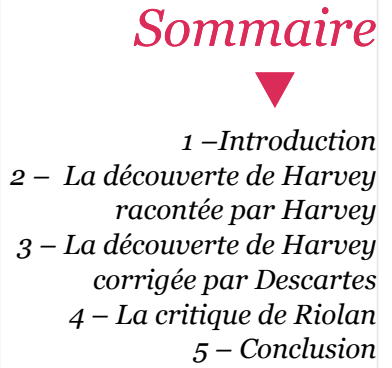

\section{1 - Introduction}

En 1628, Harvey expose la découverte de la circulation sanguine en se référant explicitement à la philosophie de la nature aristotélicienne. En 1637, dans le Discours de la méthode, Descartes adhère à la découverte de Harvey, mais en conteste le principe explicatif : il substitue à l'explication harveyenne son propre principe d'un feu sans lumière, et, du coup, remplace le mouvement circulaire aristotélicien (Aristote 1966, en particulier 20ob-208a) par un mouvement linéaire et mécanique de proche en proche. À la fois héritier et détracteur, Descartes veut débarrasser la circulation sanguine de la métaphysique aristotélicienne pour l'intégrer à la sienne. En 1649, Riolan conteste la thèse de Harvey, même s'il finit par reconnaitre la petite circulation. Il rejette l'argumentation harveyenne au profit d'une métaphysique platonico-chrétienne.

Ressaisir ces vingt années de controverses sur le sang permet de discerner, à l'occasion d'un objet historique et scientifique précis, les liens entre représentation, ontologie et métaphysique. Bien loin de se restreindre à la question factuelle de savoir si oui ou non le sang circule, ces discussions cristallisent un conflit qui se décline à trois niveaux entre Harvey, Descartes et Riolan. Elles mobilisent trois métaphysiques qui impliquent trois philosophies de la nature : l'aristotélisme, le mécanisme cartésien, le néoplatonisme renaissant. Ces trois philosophies attribuent des statuts différents au principe de vie et au cercle : la circulation comme mouvement circulaire au sens aristotélicien, le circuit comme trace du déplacement matériel des parties subtiles de proche en proche selon la loi des chocs, le cercle comme forme au sens platonicien.

Or, la controverse se justifie par la difficulté de désimbriquer ces trois dimensions de la circulation sanguine. Comment expliquer cette dissension? Bien loin de résulter d'une aberration, elle marque une irréductible pluralité des conceptions épistémologiques à l'œuvre dans les sciences, pluralité précisément due à la complexité des thèses métaphysiques, ontologiques et anthropologiques mises en jeu dans l'explication du corps vivant. Cette lecture pluraliste de la controverse admet une conception holiste des théories scientifiques non seulement en ce qu'elles intègrent une multiplicité de thèses, prérequis et définitions, mais aussi en ce qu'elles articulent plusieurs niveaux de discours. Conformément à la thèse de Quine-Duhem, on ne peut isoler un élément de la théorie : lorsque Descartes ou Riolan contestent certains aspects de la théorie de Harvey (l'âme comme principe de vie, la systole comme proprio motu ou la primauté du sang), chacun pour des raisons différentes, en réalité, chacun lui substitue un autre ensemble théorique. Cette interprétation se démarque néanmoins de l'explication kuhnienne en termes de para- 
digme puisqu'elle en conteste le critère d'incommensurabilité : les réaménagements que proposent Descartes ou Riolan à l'égard de la découverte de Harvey (Kuhn 1962, 1982 ; Bird 2008) indiquent suffisamment que la controverse ne se réduit pas à des taxinomies non congruentes et des langages incapables de communiquer entre eux. Il y a communication, mais selon des modèles de rationalité pluriels (Braunstein 2002). Dans la continuité des perspectives ouvertes par Cunningham (2003, p. 215-235), Mandressi (2013) ou Crignon (2011, 2014), cet article défend une lecture historique et critique de la controverse comme lieu de confrontation entre des rationalités diverses pour observer comment s'effectue progressivement un choix de scientificité, qui s'éprouve dans la rencontre entre des options métaphysiques, ontologiques et anthropologiques différentes ${ }^{1}$.

\section{2 - La découverte de Harvey racontée par Harvey}

La thèse de la circulation sanguine affirme trois choses : le mouvement propre du cœur est la systole ; la primauté du sang et sa chaleur constituent l'origine de la vie; l'âme réside dans le sang, sa présence se caractérisant par la chaleur, ce qui révoque la présence des esprits végétatifs, vitaux ou animaux. Ces trois sous-thèses contestent deux thèses de Galien, qui conçoit le mouvement propre du cœur comme la diastole et subordonne le cœur au foie (1854, t.1 p. 402-456). Pour Harvey, la relaxation du cœur est un mouvement secondaire, sa contraction le mouvement spontané ; le sang est premier dans l'ordre des principes de vie. En posant la primauté du sang comme partie fluide (166o, ch. 16, p. 145), Harvey ne conteste pas la thèse aristotélicienne de la primauté au cœur en tant que partie solide (Harvey 1660, ch. 17 ; Schmitt 1992, p. 131 ; Pagel 1967) ; mais, comme il l'affirme dans le De Generatione (1651), il l'accomplit (White 1986, p. 241-242).

Lorsqu'il raconte la découverte de la circulation et sa théorie sur la génération, Harvey souligne qu'il part d'abord d'une idée métaphysique qu'il applique au corps (Pagel 1976, p. 4, 1967, p. 54 sqq. ; French 2000, p. 28 ; Sawday 1995, p. 23) : si la vie est une perfection, alors les processus vitaux du sang et de la génération doivent exprimer la structure ontologique de la perfection qu'Aristote a thématisée sous la figure du cercle à partir de ses réflexions sur l'entéléchie, la cosmologie ou la vie, en particulier dans les Météorologiques (Aristote 1982 ; Martin 2002). Selon Aristote, la substance ne peut être ce qu'elle est qu'à condition de demeurer une. Il doit donc exister un mouvement éternel, continu, un et même ; or le seul mouvement qui, dans l'espace, réponde à ces critères est la circulation. Elle seule peut garantir la permanence de l'univers ou du vivant : dans leur périodicité, ils restent toujours les mêmes, parce que fondamentalement leur mouvement s'avère permanent et éternel, agissant toujours de la même manière. Le sang est principe de vie : en dépit de son déplacement et de son changement de couleur, il doit demeurer le même. Or justement, le mouvement circulaire permet de penser l'unité et l'identité dans le changement. Dans ses Exercitationes anatomicae, Harvey trace une stricte analogie entre le cosmos et le corps ; il baptise l'action du sang en référence au cycle de l'eau. Pareillement à la régénération de l'eau, les parties du corps humain reçoivent, grâce à la circulation, un sang chaud, parfait, vaporeux, spiritueux, nourrissant, qui favorise aussi bien le réchauffement, la végétation, la réfrigération ou la coagulation (Harvey 166o, p. 83-84). En ce qui concerne la génération, Harvey dresse une analogie entre, d'une part, la première goutte de sang - punctum saliens - d'où naît la vie en constituant progressivement l'oreillette droite, puis le ventricule droit, les poumons, le ventricule gauche et tout le circuit, et, d'autre part, le sperme d'où jaillit le vivant qui prolonge le cycle de la vie d'une génération à l'autre en maintenant l'espèce identique à elle-même comme à travers un cercle (Harvey 1660 ch. 4, 1662 p. 239).

En étudiant l'univers ou le corps, la philosophie naturelle s'intéresse certes à la mobilité, mais s'inscrit bien dans le prolongement d'une réflexion sur l'Être, sur son unité et les analogies (Huneman 2007 ; Hesse 1965 ; Aristote 1932, ch. 21, 1457b7, 1967, I 107b10-108a36, p. 15-16). La circulation est le mouvement propre et naturel dans le cosmos, l'espèce et le vivant. Or elle n'a d'autre but que la préservation et la permanence du monde ou de la vie sous ses deux figures, l'individu et l'espèce. Le cycle est le monde ou le sang. En reconnaissant l'impossibilité de parler univoquement de l'être ou de l'un, Aristote assume le nécessaire passage par la similitude entre les êtres en partant des analogies fondamentales de l'être. L'être se dit en plusieurs sens et diffère selon les êtres (Aristote 2010, 7Zc4 1030a21), mais quelque chose se conserve sous ces variations. D'un point de vue ontologique, l'analogie permet alors de repérer cet invariant dans la diversité des figures et processus (Aristote 1965, I 4, 643b17) : elle atteste d'une unité du réel, qui ne relève pas du genre (Aristote 2010, D 1016b37). Enfin, les deux cycles microcosmique et macrocosmique n'ont pas d'autre raison d'être que la perfection de ce qui circule. C'est leur nature, qui ne décrit pas le mobilier du monde au sens factuel que nous donnons aujourd'hui à ce terme, mais exprime la perfection de la vie et du cosmos.

Jusqu'à présent, le raisonnement de Harvey ne fait pas intervenir l'expérimentation. Certes, Harvey mobilise ses observations anatomiques et physiologiques, en particulier celles de l'embryon de poulet, lorsqu'il constate la pulsation de la première goutte de sang qui précède la formation du cœur. Mais l'ordre chronologique et hiérarchique des parties s'avère d'abord une question philosophique (White 1986, p. 244 ; French 1990). De fait, outre les éléments anatomiques, physiologiques et quantitatifs, l'argumentation repose sur une conception des degrés de perfection et une série d'ana- 
logies qui confirment l'ontologie mise en place. Roger French insiste sur le statut théorique des leçons anatomiques de Harvey (1994, p. 318, p. 66-69, p. 320) : avant de constituer une partie de la médecine, elles relèvent de la philosophie naturelle, qui cherche à comprendre la nature au triple sens à la fois aristotélicien, stoïcien et galénique de mouvementphusis, nature providence (opifex rerum) (Cicéron 2004, Livre II) et démiourgos (Galien 1821-1833 t. 3, p. 3). Son but ne se réduit pas à décrire le mouvement du cœur mais, plus radicalement, la fonction en répondant à la question du dioti-Pourquoi (French 1994, p. 96). La cause finale renvoie au concept de nature à partir de l'assimilation des corps naturels à " des œuvres et des instruments de la très grande divinité » (Harvey 1651, p. 147). Cette origine signifie que l'instrument ne peut être compris à partir de ses seules matérialité ou efficience, mais requiert de penser la forme et la finalité à l'œuvre (Bates 2000). La cause finale de la systole cardiaque consiste à rendre parfait le sang : le cœur induit le processus cyclique de régénération du sang. Expliquer la nature, c'est rendre compte de la perfection de l'Être.

Néanmoins, Harvey s'inscrit aussi dans une nouvelle conception des rapports entre expérience et science (Cook 1990, 2008) : marquée par l'étude des cas cliniques et l'observation, l'expérience prend un nouveau sens épistémologique (Dear 2008) et devient un style scientifique d'argumentation. En physique, Schaffer et Shapin ont montré comment l'expérience servait d'argument dans la controverse entre Hobbes et Boyle concernant le vide (1985, ch. 2) ; en médecine, Gianna Pomata a dégagé la spécificité du geste clinique qui se constitue sous la forme de l'observation de cas (Pomata 2010 ; Pomata \& Siraisi 2005) et Rafael Mandressi a décrit « la vérification oculaire » de l'anatomiste (2003, p. 108-109). Harvey s'inscrit dans ce renouveau qui vise à déterminer la spécificité de l'expérience médicale, désormais instruite par la pratique assidue de la dissection, voire de la vivisection. Si elles ne constituent pas - selon le récit qu'en fait Harvey - la source de sa découverte, les expérimentations et les mesures construisent un argument rhétorique, qui effectivement convainc ses contemporains.

La démonstration se déroule alors en trois temps (French 1994, p. 386) : $1^{\circ}$ l'histoire naturelle repère et décrit les structures analogiques selon les espèces, elle constitue la circulation comme objet d'observation ; $2^{\circ}$ la connaissance de l'action (proprio motu) décrit les processus à l'œuvre dans ces structures ; elle établit la circulation comme le mouvement propre de l'être ; enfin, $3^{\circ}$ la compréhension des fonctions donne la cause finale des processus ; elle explique. Néanmoins, cette découverte ne signifie pas une rupture de paradigme au sens kuhnien de révolution scientifique, comme a pu l'interpréter Grmek (1972, p. 187, 1990 ; Clericuzio 1991). Harvey ne propose pas le modèle du cœur comme une pompe ; il ne pratique pas un réductionnisme méthodologique et ne prétend à aucune mécanisation de la vie, contre l'interprétation de Charles Webster (1965; Fuchs 2001, Israel 2007, p. 11). S'il recourt effectivement à l'expérience reproductible et quantifiée, l'observation ne constitue pas un argument suffisant à ses yeux (French 1994, p. 92). De fait, les travaux historiographiques récents montrent comment Harvey concilie une enquête quantifiée et empirique des structures anatomiques avec une intuition analogique pensée en termes de degrés de perfection dans le cadre de la métaphysique aristotélicienne, conciliée avec les Écritures. S’il met effectivement en œuvre une méthode empirique, il n'en mobilise pas moins des arguments métaphysiques qui s'avèrent incontournables à l'époque pour rendre compte de l'origine de la vie. D'ailleurs, l'expérience ne désigne pas chez Harvey la reproduction d'un phénomène unique, mais plutôt une mise en perspective et une étude des rapports d'analogie entre les différentes observations (Salomon-Bayet 2008, p. 271-230). Au lieu d'une révolution, la découverte de Harvey et la controverse qu'elle suscite confirme l'idée d'une pluralité de rationalités à l'œuvre en science, selon les interprétations qu'ont pu développer Georges Canguilhem, Lorraine Daston ou Ian Hacking à l'occasion d'autres périodes ou d'autres découvertes. Le discours scientifique se caractérise par sa capacité à rectifier ses propres normes pour constituer une histoire contingente de la rationalité scientifique (Canguilhem 2009, 2002b, p. 392-412, 2002c, p. 413-428 ; Hacking 2002 ; Daston \& Galison 2007). En épistémologie de la médecine contemporaine, Michel Morange (2005, 2008, p. 35) ou Evelyne Fox Keller soulignent le danger de vouloir réduire la médecine à une " pensée unique » en montrant comment coexistent nécessairement des rationalités diverses à l'œuvre dans le vivant. Certes, le réductionnisme méthodologique a suscité de nombreuses découvertes et constitue aujourd'hui le style dominant de la science, mais il ne saurait s'arroger le monopole de la rationalité (Morange 2005, 2008, p. 38, Barberousse, Morange \& Pradeu 2009, p. 9-10 ; Fox Keller 2004). Déjà, lorsqu'il retraçait l'histoire de la découverte de la circulation sanguine et de sa réception, Fontenelle soulignait que le niveau de compréhension purement mécanique de la circulation ne suffit pas ; il faut rendre raison des structures, comprendre le plan et l'esprit général (Crignon 2011, p. 15-16).

\section{3 - La découverte de Harvey corrigée par Descartes}

À son tour, Descartes part d'une idée métaphysique. Selon la métaphore de l'arbre de la philosophie, Descartes assume en effet la nécessité de déduire les explications scientifiques d'une métaphysique. Or, son projet s'oppose d'emblée à l'aristotélisme et vise en particulier à réduire le concept aristotélicien de la vie comme mouvement propre à la seule dimension locale et quantitative (Byers 2006 ; Fuchs 2001 ; Aucante 2006). De fait, le concept de proprio motu 
se trouve au cœur de la controverse. Pour Harvey, la vie est un mouvement propre, intrinsèque, qui répond aux quatre catégories du mouvement chez Aristote. Par ailleurs, ce mouvement propre est circulaire, circularité comprise non pas seulement comme circuit, mais comme conservation dans l'altération, identité dans la différence, permanence dans le devenir. Si Descartes intègre la découverte de la circulation sanguine à sa propre explication de la vie, il conteste néanmoins la théorie harveyenne du sang : il prétend pour sa part instaurer l'explication d'un mouvement autonome à partir de la fermentation, phénomène purement matériel propagé de proche en proche par une série de chocs (French 1989). Ainsi, Descartes bascule d'une ontologie vers une autre, et le vecteur de cette transformation se situe dans le passage du concept aristotélicien de mouvement à sa définition par le seul déplacement local (Fuchs 2001, p. 130 sqq). Cette réduction est particulièrement visible au sujet de la chaleur, qui constitue pour Descartes l'ultime source du mouvement dans les corps vivants et se réduit au mouvement « des petites parties qui se remuent » en fermentant comme un feu sans lumière. Du coup, la dilatation cardiaque n'est pas le mouvement intrinsèque du cœur mais trouve sa cause prochaine dans la fermentation du sang; le principe de la vie se situe dans les seules particules du sang et des esprits agités par la chaleur du cœur (Descartes 1964-1974, XI, p. 202).

Harvey et Descartes ne parlent donc ni du même mouvement, ni de la même circulation, ni de la même vie. Pour Descartes, mouvement, circulation et vie s'avèrent purement matériels, quantitatifs et locaux (Bitbol-Hespériès 1990). En physique comme en médecine, il substitue au mouvement circulaire un mouvement linéaire; il réfute les causes finales au profit des seules causes efficientes. C'est pourquoi il n'y a pas de différence de nature entre une horloge ou une fontaine et le corps vivant (Descartes 1964-1974, VIII, p. 326, V, p. 279) ${ }^{2}$ : le sang se meut comme les aiguilles d'une horloge, certes circulairement, mais de façon mécanique et matérielle (Descartes 1964-1974, VI, p. 46-50). Ainsi la controverse entre Descartes et Harvey sur le sang marque une pluralité des rationalités. Son véritable enjeu réside dans la capacité à pouvoir discriminer entre deux représentations de la vie, deux métaphysiques et deux ontologies.

\section{4 - La critique de Riolan}

Un troisième acteur de la controverse permet de préciser la stratification des enjeux métaphysiques, ontologiques et scientifiques. Premier médecin de Marie de Médicis, doyen de la Faculté de Paris, anatomiste renommé, Jean Riolan fils (1577-1657) conteste la découverte de la circulation sanguine, même si, à la fin de sa vie, il admet la circulation pulmonaire. Il est le seul adversaire auquel Harvey prend la peine de répondre. Comme Harvey, Riolan considère l'anatomie d'abord comme une branche de la philosophie naturelle (Riolan 1626, p. 84) et la justifie en tant qu'anthropographie dans la mesure où elle constitue un moment nécessaire à la connaissance de soi. D'emblée, l'anatomie déborde largement le souci médical.

\footnotetext{
"L'anatomie se considere diversement par les Medecins, \& par les Philosophes. Les Philosophes n'ont point d'autre dessein, que de se connoistre eux-mesmes, et d'admirer l'ouvrage de leur Autheur (...). Les Medecins, outre ces intentions, ont encore celle de la faire servir à la perfection de leur Art... » (Riolan 1661, p. 1, 1626, p. 119)
}

Mais à la différence de Harvey qui ne vise pas à connaître Dieu à travers l'anatomie et n'y recourt qu'au titre de garant de l'analogie et de la perfection, l'anatomie philosophique de Riolan assume aussi une fonction théologique qui l'intègre comme un moment de la prisca theologia. Si le corps offre un miroir où contempler l'homme, le monde et Dieu, l'anatomie doit aussi confirmer les Écritures à travers la connaissance pratique du corps. Par conséquent, le corps humain doit être saisi en tant que fabrique à l'image du Créateur : avant même d'en présenter l'histoire ou la description, sa véritable connaissance requiert la philosophie et les Écritures saintes (Moraux 1981, p. 99, 113). À cet égard, Riolan assume la tradition de la Faculté de médecine de Paris dans une stricte filiation envers son père et Jean Fernel (Brockliss 1993 ; Céard 2006). À leur suite, il prétend concilier Platon, Aristote, Moïse et Galien selon une vision qui le conduit à proposer, d'une part, une lecture platonico-chrétienne de Galien ${ }^{3}$ contre une réduction naturaliste (Nutton 2001), et, d'autre part, une lecture platonisante d'Aristote. Conçue comme esprit (spiritus) selon la tradition néoplatonicienne, l'âme devient alors le principe de vie du corps (Hirai 2001, 2002, 2005, 2009). Selon l'adage classique, «le médecin commence là où le physicien (physicus) s'arrête ». Le physicien interroge le principe de vie, l'idée à l'œuvre lorsque Dieu fabrique le corps (Seguin 2010) ; le médecin s'appuie sur cette connaissance pour développer l'art thérapeutique. Cette dichotomie prouve que l'anthropographie répond d'abord à un questionnement philosophique, et que la médecine trouve son fondement dans une connaissance qui implique l'âme.

\footnotetext{
« (...) le Physicien considere l'homme seulement lors qu'il est en vie, et propre pour mettre à fin un bon nombre d'actions, tant du corps que de l'ame : il luy faut pour cela commencer l'Anatomie par le traicté de l'Ame, mais non pas le Medecin qui n'a pour son but et dernière fin, que l'homme sain ou malade. » (Riolan 1626, p. 86)
}

Alors qu'il conteste la théorie de Harvey sous la forme d'une dispute quodlibétaire, Riolan défend sa propre circulation, « nostram circulationem » (Riolan 1649, p. 6). Il oppose donc deux « doctrines » de la circulation point par point (Riolan 1649, Monitio ad lectorem, n.p.). Premièrement, il rejette 
l'analogie stricte entre la circulation cosmique et la circulation sanguine : « Le sang peut avoir un flux et un reflux alternatif dedans ces vaisseaux, mais il ne se disperse point par tout le corps, \& n'a rien de commun avec le grand mouvement circulaire. » (Riolan 1661, p. 345) Deuxièmement, il conteste le fait que le sang circule sans cesse et plusieurs fois par jour à travers tout le corps pour revenir sans cesse tout entier dans le cœur. Il propose pour sa part une circulation restreinte, en deux sens : d'abord, parce qu'elle ne s'effectue qu'au niveau des gros vaisseaux, ensuite, parce qu'elle ne concerne pas toute la masse du sang, mais seulement une partie. Contre Harvey qui pose la systole comme le mouvement propre du cœur, Riolan assigne au cœur une faculté pulsificatrice composée de la diastole et de la systole, conformément à Galien ; il maintient l'hypothèse de la communication intraventriculaire par le septum ; il rejette l'idée que le sang parte dans les extrémités par les artères et revienne par les veines ; il réfute la primauté du sang ; après avoir longtemps critiqué la circulation pulmonaire, il finit par la reconnaître tout en minimisant sa fonction (1649, p. 23), parce qu'elle ne s'effectue qu'au niveau des gros vaisseaux (1649, p.8). La circulation ne concerne donc pas toute la masse du sang, mais seulement une partie (1649, p. 22). Dans un corps sain, une seule goutte de sang circule à chaque pulsation (Riolan 1649, p. 41). Cette thèse permet en réalité de sauver la primauté du foie sur le cœur (Riolan 1661, p.196), donc la tradition galénique contre l'interprétation aristotélicienne. Troisièmement et plus fondamentalement, il rejette l'interprétation dynamique de la circulation que propose Harvey et lui substitue une interprétation galénico-néoplatonicienne statique et esthétique en tant que forme et idée du corps (« idea et species circulationis ») conformément au Timée. Ce qui vaut pour le sang vaut encore pour l'espèce : à la circulation dynamique entre les individus selon Harvey, Riolan substitue une vision formelle de l'espèce qui se conserve « par une immuable éternité » :

\footnotetext{
«par une propagation éternelle subroge un animal à la place de l'autre, un vivant au lieu d'un mort, par l'entremise de la génération (...). C'est pourquoi la génération des choses vivantes, se perpetuë par une immuable éternité, afin que ce qui ne peut durer dans le particulier des substances, se conserve à tout le moins dans le général des espèces. » (Riolan 1628, p. 364-365)
}

Aux yeux de Riolan, plus que le mouvement, c'est la figure qui révèle la nature et la perfection du corps. À la place de la référence aristotélicienne, Riolan convoque alors une autre autorité antique, Vitruve, pour établir sa représentation esthétique du corps humain parfait, tout en s'attachant à démontrer sa conformité avec les Écritures. Ainsi Riolan paraphrase la célèbre définition du De Architectura III, 1 (Vitruve 1990 ; Panofsky 1969, p. 61-68 ; Gros 2006) :

« (...) l'homme a obtenu un corps très bien compassé, et il est très vrai que la figure ronde la plus excellente de toutes les autres, est très manifestement remarquée au corps de l'homme lorsqu'il est couché sur son dos et a ses bras et jambes ouvertes : car si on vient à mettre l'une des branches d'un compas droit au nombril, et que de l'autre on tournoie alentour du corps, on touchera les extrémités des doigts, tant des pieds que des mains. (...), c'est de cette façon-là, qu'on tient le temple de Salomon avoir été bâti à l'exemple et modèle du corps humain, et la fabrique de l'Arche de Noé avoir été tirée de celle de notre corps (...) » (Riolan 1628, p. 8)

Riolan se glisse dans le topos du corps parfait vitruvien, dont il reprend toutes les caractéristiques : la double inscription dans le cercle et le carré, l'analogie avec les édifices, la valeur des chiffres 6 et 10, la médiocrité des parties et leur symétrie, le corps origine de toute autre mesure garante de beauté. La seule nouveauté consiste dans les références à l'arche de Noé et au temple de Salomon, paradigmes du bel édifice architectural. Outre la référence vitruvienne et scripturaire, Riolan se compare encore à Praxitèle, puisqu'il « ... desire découvrir ce qu'il y a de plus beau, \& de caché dans la Medecine » (1661, p.4). Il convoque aussi Polyclète et Debutades (Riolan 1628, p. 6-8 ; Tobin 1975 ; Laneyrie-Dagen 2006, p. 101 ; Pline 1985, p. 101). On retrouve encore le même geste esthétique à travers la référence au traité de Galien De anatomicis administrationibus (Galien 1821-1833, t. II, p. 218-220 ; Riolan 1661, p. 78, 1626, Praefatio n.p.) : l'anatomiste procède comme l'architecte, lorsqu'il bâtit une maison. Tous deux commencent par les fondations et l'ossature pour remonter à la superficie de l'édifice. Le corps temple de l'âme exprime l'église temple de Dieu (Riolan 1661, p. 91-93).

Bref, ces références attestent une représentation du corps qui relève plutôt du dessin et de la forme que du mouvement et de la couleur comme chez Harvey. Cette différence exprime en réalité deux traditions aristotélicienne et néoplatonicienne, dont la distinction apparaît clairement à l'occasion de l'interprétation du cercle, circulation pour l'un, forme pour l'autre. Enfin, elle marque deux rapports entre science et théologie : compatibilité pour Harvey, continuité pour Riolan qui prétend, à la suite de Galien, accomplir la théologie parfaite en saisissant la finalité des parties du corps. En reprenant à son compte l'homo bene figuratus, Riolan justifie en effet l'articulation entre anatomie, esthétique et théologie, et défend la tradition médicale de la Faculté de Paris contre les dangers de la découverte harveyenne : « après cela, je montrerai que la circulation du sang telle que je l'explique moi-même à nouveaux frais ne change rien en médecine (...) » (Riolan 1649, p. 22)

\section{5-Conclusion}

La controverse sur le sang confronte une pluralité de conceptions de la vie préconisées respectivement par Harvey, Descartes et Riolan. Cette diversité renvoie simultanément à 
plusieurs métaphysiques (aristotélicienne, cartésienne ou néoplatonicienne), plusieurs types de rapports entre philosophie naturelle et théologie (compatibilité, distinction ou conciliation), plusieurs représentations (dynamique, mécanique ou esthétique) du corps humain. Bien loin d'attester d'une incommensurabilité entre les théories, la lecture rétrospective de cette controverse met au jour les niveaux d'argumentation où se nouent les points de divergence ou d'accord. Elle manifeste un moment où la scientificité médicale assume la multiplicité des styles épistémiques de rationalité pour expliquer la complexité de la fabrique du corps et reconnaît l'impossibilité de se réduire à une pensée unique. Canguilhem, Morange ou Fox Keller ont en effet souligné la nécessité de diversifier les modes explicatifs de la vie, puisqu'elle relève de niveaux de causalité pluriels. Dans cette quête, la connaissance de la vie mobilise tout autant des postures métaphysiques, des traditions philosophiques et médicales, des expériences, d'autres savoirs tels que les météorologiques, la physique mécanique ou l'esthétique. La rupture qu'induit cette découverte ne concerne donc pas seulement le fait de la circulation, mais plus substantiellement une reconfiguration du savoir dans ses principes, sa méthode et son objet.

Plutôt qu'en termes de révolution, Andrew Cunningham analyse la modernité médicale comme une réforme : Luther a réformé la théologie, les médecins réforment la médecine en interrogeant le fondement du savoir. Bien loin de couper toute référence antique, ils assument leur filiation et transforment l'usage des Anciens en les important dans des schèmes décisifs pour la Modernité. Claire Crignon propose la catégorie de refonte pour indiquer une réorganisation profonde du savoir (Canguilhem 2002a ; Crignon 2011, 2014). L'ancienne théorie ne constitue pas un simple obstacle épistémologique, mais se trouve reconfigurée par l'expérience et la mise en place d'une nouvelle représentation de la vie. Rafael Mandressi étudie le rôle essentiel de la médecine dans le réaménagement des savoirs de la première Modernité (Frühe Neuzeit). Il n'y a pas une ligne de partage unique et fixe entre modernes et anciens, vitalistes et mécanistes, aristotéliciens et anti-aristotéliciens ; la controverse réorganise la rationalité scientifique non seulement en tant que théorie mais aussi dans ses implications métaphysiques, ontologiques ou anthropologiques. La suite de l'histoire semble le prouver : elle n'a pas consisté à choisir Harvey contre Descartes ou Riolan, mais à concilier des systèmes $a$ priori contraires, à éliminer certaines thèses et à configurer à partir des pièces du puzzle une nouvelle figure de la science médicale moderne.

Remerciement

Je remercie Pierre Olivier Méthot pour ses remarques.

\section{RÉFÉRENCES}

ARISTOTE. 1932. Poétique. Trad. par J. Hardy. Paris : Belles Lettres.

ARISTOTE. 1965. Les parties des animaux. Trad. par P. Louis.
Paris : Belles Lettres.

ARISTOTE. 1966. Physique. Trad. par H. Carteron. Paris : Belles Lettres.

ARISTOTE. 1967. Topiques, Livres I-IV. Trad. par J. Brunschwig. Paris : Belles Lettres.

ARISTOTE. 1982. Météorologiques. Trad. par P. Louis. Paris : Belles Lettres.

ARISTOTE. 2010. Les Métaphysiques. Trad. par A. de Muralt. Paris : Belles Lettres.

AUCANTE, Vincent. 2006. La philosophie médicale de Descartes. Paris : PUF. Livre.

BARBEROUSSE, Anouk, MORANGE, Michel, PRADEU, Thomas (dir.). 2009. Mapping the future of biology. Evolving concepts and theories. Springer. Livre.

BATES, Don. 2000. Machina ex Deo : William Harvey and the Meaning of Instrument. Journal of the History of Ideas, 61(4), 577-593. Article.

BIRD, Alexander. 2008. Incommensurability naturalized. In SOLER, L., SANKEY, H., HOYNINGEN-HUENE, P. (dir.). Rethinking scientific change and theory comparison : Stabilities, Ruptures, Incommensurabilities. Dordrecht : Springer. 21-39. Chapitre.

BITBOL-HESPERIES, Annie. 1990. Le principe de vie chez Descartes. Paris : Vrin.

BRAUNSTEIN, Jean-François. 2002. Bachelard, Canguilhem, Foucault. Le « style français » en épistémologie. In WAGNER, Pierre (dir.). Les philosophes et la science. Paris : Gallimard. 920963.

BROCKLISS, Laurence. 1993. Seeing and Believing : Contrasting attitudes towards observation. In BYNUM William F., PORTER Roy (dir.). Medicine and the Five senses. Cambridge : Cambridge University Press. 69-84.

BYERS, Sarah. 2006. Life as 'self-motion' : Descartes and 'the Aristotelians' on the soul as the life of the body. The review of metaphysics, 59(4), 723-755.

CANGUILHEM, Georges. 2002a [1968]. L'homme de Vésale dans le monde de Copernic. In Études d'histoire et de philosophie des sciences concernant les vivants et la vie. Paris : Vrin. 27-35.

CANGUILHEM, Georges. 2002b [1978]. Puissance et limite de la rationalité en médecine. In Études d'histoire et de philosophie des sciences. Paris : Vrin. 392-412 ;

CANGUILHEM, Georges. 2002c. Le statut épistémologique de la médecine. In Études d'histoire et de philosophie des sciences. Paris : Vrin. 413-428.

CANGUILHEM, Georges. 2009 [1977]. Idéologie et rationalité dans l'histoire des sciences de la vie. Paris : Vrin.

CÉARD, Jean. 2006. La physiologie de Fernel : l'anatomie et la physiologie, ou la géographie et l'histoire du corps humain. In ZINGUER, Ilana, MARTIN, Isabelle (dir.). Théâtre de l'anatomie et corps en spectacle. Berne : Peter Lang. 49-62.

CICÉRON. 2004. La nature des dieux. Trad. par Clara Auvray-Assayas. 2e éd. Paris : Belles Lettres.

CLERICUZIO, Antonio. 1991. Review : La première révolution biologique: Reflexions sur la physiologie et la medecine du XVIIe siècle. Medical history, 35(4), 1991. 456-457. Article.

COOK, Harold J. 1990. The New Philosophy and Medicine in se- 
REVUE

DE LA SOCIÉTÉ

DE PHILOSOPHIE

DES SCIENCES
LA CIRCULATION SANGUINE

COMME PIERRE DE TOUCHE : Harvey, Riolan, Descartes
venteenth-Century England. In LINDBERG D. and WESTMAN R. (dir.). Reappraisal of the Scientific Revolution. Cambridge : Cambridge University Press. 397-436.

COOK, Harold J. 2008 [2006]. Medicine. In PARK, Katherine, DASTON, Lorraine (dir.). The Cambridge History of Science. Early Modern Science, vol.3. Cambridge : Cambridge University Press. 407-435. Chapitre.

CRIGNON, Claire. 2011. La découverte de la circulation sanguine : révolution ou refonte ? Gesnerus 68(1), 5-25.

CRIGNON, Claire. 2014. Révolution anatomique et révolution cosmologique : quelques réflexions à partir de la lecture de « l'homme de Vésale dans le monde de Copernic ». Revue de Métaphysique et de morale, 2(82), 167-195. Article.

CUNNINGHAM, Andrew. 2003 [1997]. The anatomical Renaissance. The resurrection of the anatomical projects of the Anciens. Aldershot: Ashgate. Livre.

DASTON, Lorraine, GALISON, Peter. 2007. Objectivity. New York : Zone books.

DEAR, Peter. 2008 [2006]. The meaning of experience. In PARK, K., DASTON L. (dir.). The Cambridge History of Science. Early Modern Science, vol.3. Cambridge : Cambridge University Press. 106-131. Chapitre.

DESCARTES, R. 1964-1974. Euvres de Descartes. ADAM, C., TANNERY, P. (dir.), nouv. prés. par B. Rochot et P. Costabel. 11 vol. Paris : Vrin. Livre.

FLEMING, Donald. 1955. Harvey and pulmonary circulation. Isis, 46(4), 319-327. Article.

FOX KELLER, Evelyn. 2004. Expliquer la vie : modèles, métaphores et machines en biologie du développement. Trad. par S. Schmitt. Paris : Gallimard.

FRENCH, Roger. 1989. Harvey in Holland : circulation and the Calvinists. In FRENCH, R., WEAR, A. (dir.) The medical revolution of the seventeenth century. Cambridge : Cambridge University Press. 46-86. Chapitre.

FRENCH, Roger. 1990. Natural Philosophy and anatomy. In CEARD, Jean, FONTAINE, M., MARGOLIN, J.C. (dir.). Le corps à la Renaissance. Paris : Aux Amateurs de Livres. 447-460.

FRENCH, Roger. 1994. William Harvey's natural Philosophy. Cambridge : Cambridge University Press. Livre.

FRENCH, Roger. 2000. The Languages of William Harvey's Natural Philosophy. In Ancients and Moderns in the Medical Sciences. Aldershot, Ashgate. 24-51. Chapitre.

FUCHS, Thomas. 2001. The mechanization of the heart : Harvey and Descartes. Rochester : Rochester University Press.

GALIEN. 1821-33. Opera omnia. KÜHN, C.G. (dir.). Leipzig. Livre. GALIEN. 1854-1856. Euvres anatomiques, physiologiques et médicales. DAREMBERG, C. (dir.). 2 vol. Paris : J.B. Baillière.

GRMEK, Mirko, D. 1972. A survey of the mechanical interpretations of Life. In BRECK, Allen, D., YOURGRAU, Wolfgang (dir.). Biology, History, and Natural Philosophy. New York: Plenum Press. Chapitre.

GRMEK, Mirko, D. 1990. La première révolution biologique. Réflexions sur la physiologie et la médecine du dix-septième siècle. Paris : Payot.

GROS, Pierre. 2006. Vitruve et la tradition des traités d'architec- ture. Fabrica et ratiocinatio. École française de Rome. Livre. HACKING, Ian. 2002. Historical ontology. Cambridge Mass. : Harvard University Press.

HARVEY, William. 1651. Exercitationes de Generatione Animalium. Quibus accedunt quaedam De Partu, de Membranis ac humoribus Uteri, \& de Conceptione. Londres : Du Gardian. Livre. HARVEY, William. 1660. Exercitationes anatomicae de motu cordis et sanguinis circulatione. Rotterdam : Arnold Leers. Livre.

HARVEY, William. 1662. Exercitationes de Generatione Animalium. Quibus accedunt quaedam De Partu, de Membranis ac humoribus Uteri, \& de Conceptione. Autore Guilielmo Harveo Anglo... Amstelodami. Livre.

HESSE, Mary. 1965. Aristotle's logic of analogy. The Philosophical Quaterly, 15(61), 328-340. Article.

HIRAI, Hiroshi. 2001. Paracelsisme, néoplatonisme et médecine hermétique dans la théorie de la matière de Joseph Duchesne à travers son Ad veritatem hermeticae medicinae (1604). Archives internationales d'histoires des sciences, 51, 9-37.

HIRAI, Hiroshi. 2002. La fortune du concept de semence de Marsile Ficin au seizième siècle. Accademia : Revue de la Société Marsile Ficin, 4, 109-132.

HIRAI, Hiroshi. 2005. Alter Galenus : Jean Fernel et son interprétation platonico-chrétienne de Galien. Science and Medicine, 10, 1-35. Article.

HIRAI, Hiroshi. 2009. Lecture néoplatonicienne d'Hippocrate chez Fernel, Cardan et Gemma. In VONS, Jacqueline (dir.). Pratique et pensée médicales à la Renaissance. Paris : De Boccard. 91-110.

HUNEMAN, Philippe. 2007. Sur la conception aristotélicienne de l'analogie. In DURAND-RICHARD, Marie-José (dir.). L'usage opératoire de l'analogie en sciences. Paris : L'Harmattan. 30-60. ISRAEL, Jonathan. 2007. Radical Enlightenment and the Medical Revolution of the Late XVIIth and XVIIIth centuries. In GRELL, Ole Peter, CUNNINGHAM, Andrew (dir.). Medicine and Religion in Enlightenment Europe. Ashgate.

KUHN, Thomas. 1962. The Structure of Scientific revolution. Chicago : University of Chicago Press.

KUHN, Thomas S. 1982. Commensurability, communicability, comparability. PSA: Proceedings of the Biennial Meeting of the Philosophy of Science Association, 2, 669-688. Article.

LANEYRIE-DAGEN, Nadeije. 2006. Lïnvention du corps. La représentation de l'homme du Moyen Âge à la fin du XIXe siècle. Paris : Flammarion.

MANDRESSI, Rafael. 2003. Le regard de l'anatomiste. Paris : Seuil. Livre.

MANDRESSI, Rafael. 2013. Médecine et discours sur l'homme dans la première modernité. Revue de synthèse, 134(4), 511-536. Article.

MARTIN, Craig. 2002. Francisco Valles and the Renaissance Reinterpretation of Aristotle's Meteorologica IV as a Medical Text. Early Science and Medicine, 7(1), 1-30. Article.

MORANGE, Michel. 2005. Les secrets du vivant : contre la pensée unique en biologie. Paris : Odile Jacob.

MORANGE, Michel. 2008. A quoi sert l'histoire des sciences ?Versailles : ed. Quae. Livre. 
MORAUX, Paul. 1981. Galien comme philosophe : la philosophie de la nature. In NUTTON, Vivian (dir.). Galen: Problems and Prospects. London : The Wellcome Institute. 87-116.

NUTTON, Vivian. 2001. God, Galen and the Depaganization of Ancient Medicine. In BILLER, Peter, ZIEGLER, Joseph (dir.). Religion and Medicine in the Middle Ages. York : York Medieval Press. 17-32.

PAGEL, Walter. 1967. William Harvey's Biological Ideas. Selected Aspects and Historical Backgrounds. Basel-New York : Karger Publishers.

PAGEL, Walter. 1976. New light on William Harvey. Basel-New York : Karger Publishers.

PANOFSKY, Erwin. 1969. L'évolution d'un schème structural. L'histoire de la théorie des proportions humaines conçue comme un miroir de l'histoire des styles. In L'ouvre d'art et ses significations. Essais sur les arts visuels. Traduit par B. Teyssèdre. Paris : Gallimard.

PLINE l'Ancien. 1985. Histoire naturelle. Paris : Belles Lettres.

POMATA, Gianna. 2010. Sharing Cases: The Observationes in Early Modern Medicine. Early Science and Medicine, 15(3). 193236. Article.

POMATA, Gianna, SIRAISI, Nancy. 2005. Historia: Empiricism and Erudition in Early Modern Europe. Cambridge : MIT Press.

RIOLAN, Jean. 1626. Anthropographia, et osteologia. Omnia recognita, triplo auctiora et emendatiora ex proprïs, ac nouis cogitationibus, et obseruationibus. Cum duplici indice, vno capitum, \& praecipuorum articulorum : altero rerum \& verborum insigniorum copiosissimo. Parisijs : ex officina Dionysij Moreau.

RIOLAN, Jean. 1628. Les œuvres anatomiques de Monsieur Jean Riolan, reveues et augmentées d'une cinquième partie en ceste édition, le tout rangé, divisé, noté et mis en françois par M. Pierre Constant. Paris : Denys Moreau.

RIOLAN, Jean. 1649. Opuscula anatomica nova, quae nun in lucem prodeunt. Instauratio magna Physicae et Medicinae per no-

\section{HISTORIQUE}

Article initialement soumis le 31 janvier 2015.

Article révisé soumis le 1 juillet 2015.

Article accepté le 28 août 2015

SITE WEB DE LA REVUE

sites.uclouvain.be/latosensu/index.php/latosensu/index

ISSN 2295-8029

DOI http://dx.doi.org/10.20416/lsrsps.v3i1.243 vam doctrinam de motu circulatorio sanguinis in corde. Londres : Milonis Flescher.

RIOLAN, Jean. 1651. Curieuses recherches sur les escholes en medecine de Paris et de Montpellier. Paris : Gaspar Meturas.

RIOLAN, Jean. 1661. Manuel anatomique et pathologique, ou Abrégé de toute l'Anatomie et des Usages que l'on peut en tirer pour la connoissance, \& pour la guerison des Maladies. Paris : Gaspar Meturas.

SALOMON-BAYET, Claire. [1978] 2008. Linstitution de la science et l'expérience du vivant. Méthode et expérience à l'Académie Royale des Sciences, 1666-1793. Paris : Flammarion.

SAWDAY, Jonathan. 1995. The Body Emblazoned. Dissection and the Human Body in Renaissance Culture. London \& New York : Routledge. Livre.

SCHAFFER, Simon, SHAPIN, Steven. 1985. Leviathan and the Air-pump : Hobbes, Boyle and the experimental life. Princeton : University Press of California.

SCHMITT, Charles B. 1992 [1983]. Aristote et la Renaissance. Paris : PUF. Livre.

SEGUIN, Maria Susana. 2010. Du discours sur la nature au langage scientifique. Revue Fontenelle, 6-7, 311-324.

TOBIN, Richard. 1975. The Canon of Polykleitos. American Journal of Archaelogy, 79, 307-321. Article.

VITRUVE. 1990. De l'Architecture, livre I. Trad. par Ph. Fleury. Paris : Les Belles Lettres.

VITRUVE. 1990. De l'Architecture, livres II, III, IV. Trad. par Pierre Gros. Paris : Les Belles Lettres.

WEBSTER, Charles. 1965. William Harvey's conception of the heart as a pump. Bulletin of the History of Medicine, 39, 508-517. WEBSTER, Charles. 2002 [1975]. The great instauration. Science, Medicine and Reform 1626-166o. Bern : Peter Lang.

WHITE, John S. 1986. William Harvey and the primacy of the blood. Annals of science, 43(3), 239-255. Article.

CONTACT ET COORDONNÉES

Sarah Carvallo

École Centrale de Lyon

UMR 5317. ENS Lyon

36 avenue Guy de Collongue

69134 Ecully Cedex

France

sarah.carvallo@ec-lyon.fr

: $\mathbf{S P S} \quad$ www.sps-philoscience.org

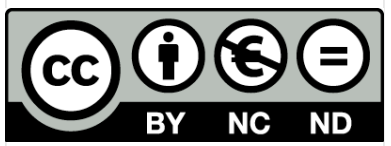

